\title{
Model-based Angiogenic Inhibition of Tumor Growth using Modern Robust Control Method ${ }^{\star}$
}

\author{
Annamária Szeles*, Johanna Sápi *, Dániel A. Drexler*, \\ István Harmati *, Zoltán Sápi **, Levente Kovács * \\ * Department of Control Engineering and Information Technology, \\ Budapest University of Technology and Economics, Budapest, Hungary \\ (e-mails: szeles.annam@gmail.com, \{sapi, drexler, harmati, \\ lkovacs\}@iit.bme.hu) \\ ** 1st Department of Pathology and Experimental Cancer Research, \\ Semmelweis University, Budapest, Hungary \\ (e-mail: sapi.zoltan.dr@gmail.com)
}

\begin{abstract}
Cancer is one of the most destructive and lethal illnesses of the modern civilization. In the last decades, clinical cancer research shifted towards molecular targeted therapies which have limited side effects in comparison to conventional chemotherapy and radiation therapy. Anti-angiogenic therapy is one of the most promising cancer treatment methods. The dynamical model for tumor growth under angiogenic stimulator/inhibitor control was posed by Hahnfeldt et al. (1999), and it was investigated and partly modified many times. In this paper, a modified version of the originally published model is used in order to describe a continuous infusion therapy. To generalize individualized therapies a robust control method is proposed using $\mathcal{H}_{\infty}$ methodology. Uncertainty weighting functions are determined based on the real pathophysiological case and simulations are performed on different tumor volumes to demonstrate the robustness of the proposed method.
\end{abstract}

Keywords: biomedical systems, robust control, H-infinity optimization, tumor therapy

\section{INTRODUCTION}

Cancer is in the front line of lethal illnesses which demands the highest number of human lives in modern societies - 7.6 million people worldwide died from cancer in 2008 (World Health Organization (2012)). Because of that, research and treatment of cancer is a very important mission for medicine. Beside classical methods there are a lot of new therapies, based on mathematical models, where human body works as a complex system.

Antiangiogenic therapy represents a relatively new targeted molecular therapy, which had come up in the last decade and its aim is to inhibit angiogenesis (creating a vascular system of the tumor), which leads to the death of the tumor over the critical volume. (Pluda (1997)).

The main advantage of antiangiogenic therapy to conventional (like chemotherapy) treatments is that tumor cells can not become resistant towards the antiangiogenic drugs and antiangiogenic therapy can be used with nontoxic

\footnotetext{
* This work was supported in part by the National Office for Research and Technology (NKTH), Hungarian National Scientific Research Foundation grants OTKA K71762 and K82066. It is connected to the scientific program of the "Development of qualityoriented and harmonized $\mathrm{R}+\mathrm{D}+\mathrm{I}$ strategy and functional model at BME" project, supported by the New Hungary Development Plan (Project ID: TMOP-4.2.1/B-09/1/KMR-2010-0002). Levente Kovács was also supported by the János Bolyai Research Scholarship of the Hungarian Academy of Sciences.
}

concentrations (Kerbel (1997); Qian et al. (2011)). As the idea is not to eliminate the whole tumor, but to control and keep it in a given state, model-based treatment, and hence the use of control theory is an ideal choice. Moreover, robustness is also important to be able to generalize the treatment.

Linear $\mathcal{H}_{\infty}$ control syntheses are promising methods on the palette of the robust control systems. This postmodern technique dates back to around two decades (Doyle et al. (1989)). Progressively it gains ground by the more and more powerful computational soft- and hardware (Zhou (1996); Gu et al. (2005)). One of the biggest advantages of these methodologies (beyond the well defined mathematical backgrounds) might be the robustness itself. Robustness against model mismatches, against disturbances.

This current paper is intended to discuss a model-based angiogenic inhibition of tumor growth by use of $\mathcal{H}_{\infty}$ methodology.

The paper is organized as follows. Section 2 gives a short review of conventional and targeted molecular therapies, while Section 3 presents the tumor model of our investigations. In Section 4 the $\mathcal{H}_{\infty}$ design is summarized, while simulation results are presented in Section 5. Finally, the paper ends with the conclusions and future work aspects in Section 6. 


\section{BIOMEDICAL BACKGROUND}

Conventional cancer therapies (surgery, chemotherapy, radiotherapy) fight directly against the tumor cells. Surgery is the oldest modality of cancer therapy, which treats cancer by removing the gross lesion. Surgical intervention is most effective in the treatment of localized primary tumor disease.

Chemotherapy (chemical treatment) is a classical treatment of cancer with anticancer drugs. Choosing the best suited anticancer drug for a patient is based on the type of cancer, the patient's age and health, and other drugs the patient is taking. Most chemotherapy drugs interfere with the ability of cells to grow or multiply; hence these treatments are most effective against rapidly growing cells (The Free Dictionary (2012)), but it has effects on certain healthy cells of the patient as well. In addition, tumor cells can become resistant towards the drug used in chemotherapy, which makes the usage of new drugs necessary.

Radiotherapy is the medical use of ionizing radiation, primarily high-energy photons (gamma rays and x-rays) and charged particles (electrons). Radiation is randomly deposited within the cell and results DNA damage. This damage is primarily manifested by the loss of cellular reproductive integrity. Tumor cells are dividing rapidly, so the functional disorder resulted by radiotherapy is leading to cellular death. Similar to chemotherapy, radiation therapy also has effects on healthy cells; therefore, in chemoand radiotherapy side effects are significant.

Summarizing conventional cancer therapies (Holland and Frei (2003)): surgery kills all the excised cells (zero-order kinetics), in contrast to using chemotherapy or radiation therapy where only a fraction of tumor cells are killed (first-order kinetics). When a cancer has been removed by surgery, chemotherapy or radiotherapy may be used to keep the cancer from coming back (adjuvant therapy).

Beside conventional therapies, a new approach is represented by model-based therapies, called targeted molecular therapies (TMTs). TMTs work by different ways (Li et al. (2012)) and in contrast to chemo- and radiotherapy, fight specifically against certain cancer mechanisms (for example antiangiogenic inhibitors interfere with cell growth signaling or stimulate the immune system to destroy specific cancer cells). By focusing on molecular and cellular changes that are specific to cancer, targeted molecular therapies may be more effective than conventional treatments, and less harmful to normal cells (have limited side effects). The aim of targeted molecular therapies is not to eliminate the whole tumor, but to control the tumor into a given state and keep it there.

A promising field in TMTs is antiangiogenic therapy, which had come up in the last decade (Pluda (1997)). As rapidly dividing tumor cells need lots of oxygen, when proliferation begins, small sized tumor can pick up oxygen from near capillaries. After a certain size (1-2 mm diameter) tumor development stops, because a part of the tumor gets too far from capillaries and cannot pick up enough oxygen. Hence, the tumor needs its own blood vessels - the process of forming new blood vessels is called angiogenesis (Gotink and Verheul (2010); Kopper and Tímár (2007)).
Angiogenesis occurs normally in the human body at specific times in embryonic development and growth (for ex. a developing child in a mother's womb must create the vast network of arteries, veins, and capillaries that are found in the human body). Angiogenesis also takes place in adults (Kleinsmith et al. (2010)), although it is a relatively infrequent event (in case of high altitude (low oxygen concentration), regeneration of tissue during wound healing and in women during certain phases of the menstrual cycle). In such cases, angiogenesis starts due to typical molecular triggers and ends when the necessary processes are completed.

However, tumors can break through this precise control. The aim of antiangiogenic cancer therapy is to prevent tumors from forming new blood vessels, because without angiogenesis tumor growth is inhibited. Several angiogenic inhibitors are known in medical practice, for ex. endostatin (O'Reilly et al. (1997)) or bevacizumab (Ellis and Haller (2008)). Contrary to conventional treatments, using antiangiogenic therapy, tumor cells cannot become resistant towards the antiangiogenic drugs (this is achieved by antiangiogenic therapy as it is directed against the tumor supplying blood vessels) Kerbel (1997) and antiangiogenic therapy can be used with nontoxic concentrations (Qian et al. (2011)). However, due to high costs of the antiangiogenic drugs, their optimal usage is preferred which gives an ideal field for model-based and optimal control theory.

\section{DYNAMICAL MODEL OF TUMOR GROWTH}

At the Harvard Medical University, Hahnfeldt et al. (1999) comprised the complete model formulation for tumor growth control under the actions of angiogenic stimulation and inhibition. In their experience, mice were injected with Lewis lung carcinoma cells.

From the original model one can derive the following second-order system (Hahnfeldt et al. (1999)):

$$
\begin{aligned}
\dot{x}_{1} & =-\lambda_{1} x_{1} \ln \left(\frac{x_{1}}{x_{2}}\right) \\
\dot{x}_{2} & =b x_{1}-d x_{1}^{2 / 3} x_{2}-e x_{2} u \\
y & =x_{1}
\end{aligned}
$$

where $x_{1}$ is the tumor volume $\left(\mathrm{mm}^{3}\right), x_{2}$ is the supporting vasculature volume $\left(\mathrm{mm}^{3}\right)$, and $u$ is the serum level of the inhibitor $(\mathrm{mg} / \mathrm{kg})$. The parameters characteristic for the Lewis lung carcinoma and the mice used in the experiment are: $\lambda_{1}=0.192\left(\right.$ day $\left.^{-1}\right)$ describing the speed of the Gompertzian growth, $b=5.85\left(\right.$ day $\left.^{-1}\right)$ describing the stimulating effect of the tumor on angiogenesis, $d=0.00873\left(\right.$ day $\left.^{-1} \mathrm{~mm}^{-2}\right)$ describing inherent angiogenic inhibition effects, and the parameter characteristic for the applied inhibitor (endostatin) is: $e=0.66\left(\right.$ day $\left.^{-1}(\mathrm{mg} / \mathrm{kg})^{-1}\right)$ describing the effect of angiogenic inhibition on the supporting vasculature. These numerical values of the model parameters will be used throughout the paper, both at the controller design and simulation phases.

Fig. 1. shows the tumor growth slowdown and asymptotic limit $\left(17340 \mathrm{~mm}^{3}\right)$ of Lewis lung tumor size that would hypothetically be reached if this size of tumor were compatible with the life of the animal (see upper part 

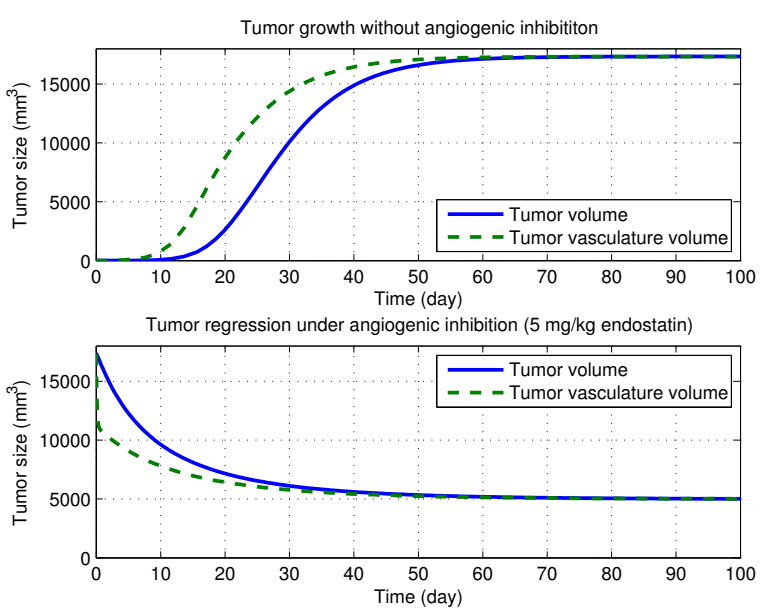

Fig. 1. Tumor growth without and under angiogenic inhibition

of Fig. 1, being equivalent of an open-loop therapy. This is also equivalent to a lethal steady-state case). Under anti-angiogenic treatment (constant $5 \mathrm{mg} / \mathrm{kg}$ endostatin), initiated from the largest tumor volume, tumor size is projected to plateau as a result of parallel plateauing of available vascular support. Angiogenic inhibitors act to generate lower plateau points (see lower part of Fig. 1, equivalent to a model-based therapy, but yet without control strategy).

Since the aim of this work is to design robust control for the system, deriving a linear model is necessary, as the $\mathcal{H}_{\infty}$-control design requires a linear nominal model. The linear model is acquired by working point linearization. The symbolic form of the linearization is:

$$
\begin{aligned}
& \dot{x}_{1}=\left(-\lambda_{1} \ln \left(\frac{x_{10}}{x_{20}}\right)-\lambda_{1}\right) x_{1}+\lambda_{1} \frac{x_{10}}{x_{20}} x_{2} \\
& \dot{x}_{2}=\left(b-\frac{2}{3} d x_{10}^{-\frac{1}{3}} x_{20}\right) x_{1}+\left(-d x_{10}^{\frac{2}{3}}-e u_{0}\right) x_{2}-e x_{20} u
\end{aligned}
$$

$$
y=x_{1} .
$$

The non-trivial equilibrium points of the linearized model can be calculated from (4), i.e. $x_{10}=x_{20}$. The assessed working point is $x_{10}=x_{20}=100 \mathrm{~mm}^{3}, u_{0}=5 \mathrm{mg} / \mathrm{kg}$, (Drexler et al. (2011)). The eigenvalues of the linearized system are $p_{1}=0.1132$ and $p_{2}=-3.7933, p_{1}$ is on the right-half plane, thus the system is unstable, corresponding to the pathophysiological case. After evaluating the controllability and the observability criteria, one gets that the system is controllable and observable.

\section{4. $\mathcal{H}_{\infty}$ CONTROL DESIGN}

The objective of the problem is to design a linear, robust controller, which achieves good tracking property, takes into account the model uncertainties and limits the magnitude of the control input and the disturbance. The signals of the system are the following: $r$ is the reference, $u$ is the control input, $y$ is the output, $n$ is the measurement noise, $e$ is the modeling error, $d$ is the disturbance caused by the uncertainty of the model, $z_{u}$ is the penalized control input, whereas $z_{e}$ is the deviation of the output from the required one (Fig. 2).
The closed-loop system includes the feedback structure of the nominal model $G_{n}$ and the two-degree controller $K$, which is portioned in two parts: $K_{y}$ is the feedback part to meet the requirements of internal and robust stability, disturbance rejection, measurement noise attenuation, and sensitivity minimisation, while $K_{r}$ is the prefilter part, which optimises the response of the overall system to the command input such that the output of the system would be near to that of the chosen ideal system.

The input multiplication uncertainty $W_{\text {unc }}$ takes into consideration the differences between the nominal model and the real plant. The weighting function $W_{n}$ stands for the limitation of sensor noise. The limitation of the control input is achieved by the weighting function $W_{u}$ which penalizes larger deflections. The model matching function $T_{i d}$ describes the ideal transfer function of the plant. Since the designed controller should effect tumor regression even in the worst case, the reference model, $T_{i d}$ describes fast regression from the maximal tumor volume predicted by the model. The weighting function $W_{\text {perf }}$ penalizes tracking.

Starting from the formal definition of the multiplicative uncertainty, parametric sensitivity was performed on the nonlinear model to determine $W_{\text {unc }}$. The idea was partially adapted and modified from Liu and Tang (2008) and Kovács et al. (2011). The idea is to take uncertain parameters in the nonlinear model. After taking every single extremal combination of the parameters using a gridding technique, linearization is performed. In our case a $\{ \pm 5 \%, \pm 2.5 \%, 0 \%\}$ grid was used for the variability of the Lewis lung carcinoma parameters $(b, d)$, and a $\{ \pm 10 \%, \pm 5 \%, 0 \%\}$ grid was used for vascular inactivation rate $(e)$.

Finally, the frequency content of the perturbed and linearized model is compared, and relative difference is computed,

$$
W_{r e l}(\omega)=\left|\frac{G_{p}(\omega)-G_{n}(\omega)}{G_{n}(\omega)}\right|
$$

where $G_{p}$ stands for the perturbed model and $G_{n}$ for the nominal one.

The obtained uncertainty weighting function, $W_{u n c}$ should work as a high pass filter to reduce disturbance at low frequency, and to avoid strong restrictions at high frequency. Sensor-noise, as a wide-band signal, can be modeled with a constant value. During the design process, $W_{n}$ anticipates $5 \%$ measurement noise for volume measurements. The weight on endostatin inlet, $W_{u}$ is defined to be constant over the entire frequency range and with a magnitude equal to the inverse of the maximal possible inlet $(1 / 100 \mathrm{mg} / \mathrm{kg})$, because of physiological reasons (since high doses are not desirable). $W_{\text {perf }}$ should be large in frequency range where small errors are desired and small where larger errors can be tolerated. The chosen weighting functions can be seen in Fig. 3 .

The generalized structure of $\mathcal{H}_{\infty}$ control design is formulated in $\Delta-\mathrm{P}-\mathrm{K}$ structure, (Fig. 4). As signals, $w^{*}=\left[\begin{array}{rr}r & n\end{array}\right]$ denotes the external inputs, $z^{*}=\left[\begin{array}{ll}z_{e} & z_{u}\end{array}\right]$ represents the output signals to be minimized or penalized. The closedloop function $M$ can be derived as the lower linear fractional transformation of the pair $(P, K)$. 


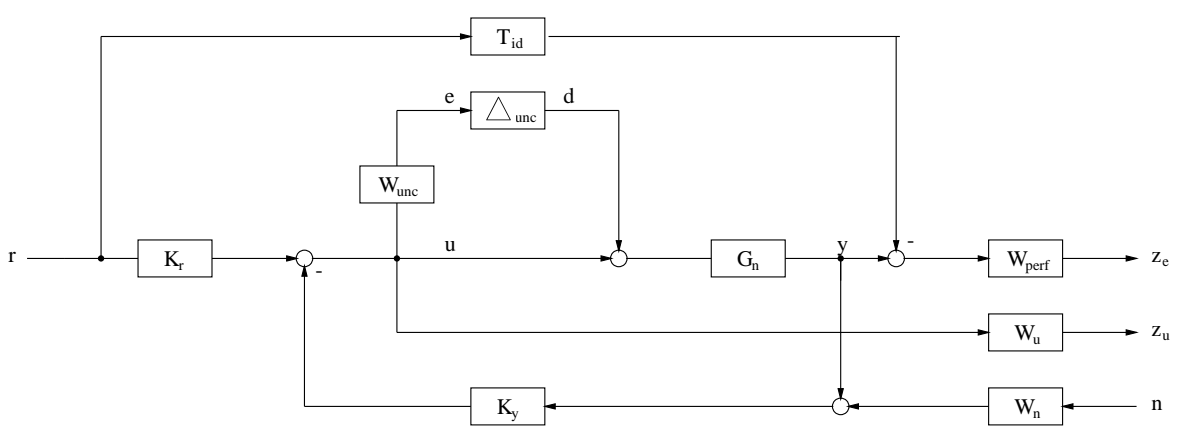

Fig. 2. The closed-loop interconnection structure

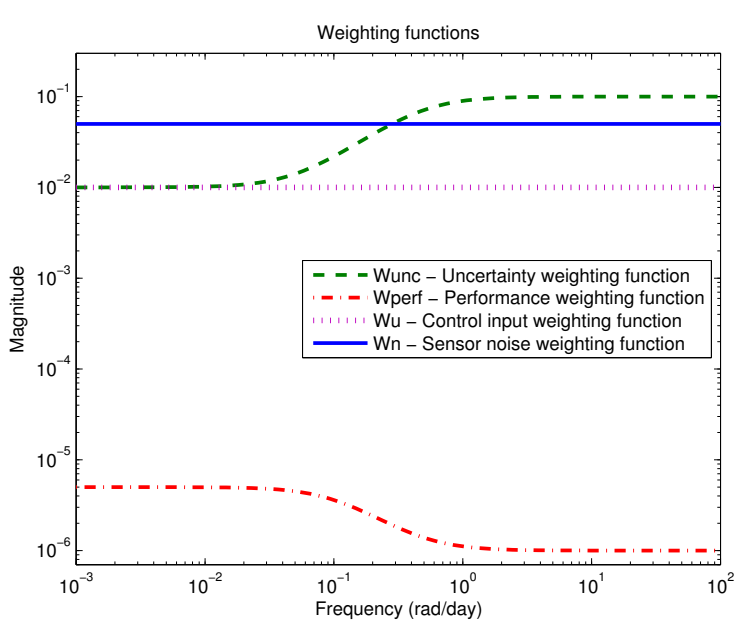

Fig. 3. Weighting functions in frequency domain

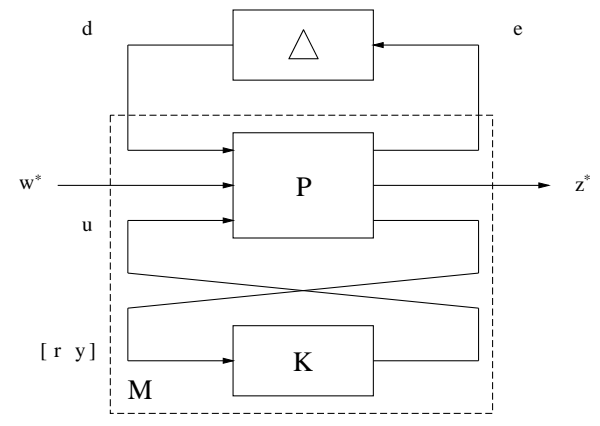

Fig. 4. The generalized $\Delta-\mathrm{P}-\mathrm{K}$ structure

The aim of the suboptimal design is to find a stabilising controller $K$ such that the $\mathcal{H}_{\infty}$-norm of the closed-loop function is less than a given positive number, Zhou (1996), i.e.,

$$
\|M\|_{\infty}=\left\|\mathcal{F}_{\ell}(P, K)\right\|_{\infty}<\gamma
$$

The scope of the $\mathcal{H}_{\infty}$ controller design is to guarantee robust performance of the system. This can be realized by fulfilling the conditions of robust stability and nominal performance. To guarantee nominal stability the system must be internally stable, which means that the created transfer function is stable from all inputs to all outputs. The robust stability is achieved by fulfilling the following condition:

$$
\left\|M_{11}\right\|_{\infty}<1
$$

The nominal performance is achieved if the performance objective is satisfied:

$$
\left\|M_{22}\right\|_{\infty}<1 .
$$

As a result, the conditon of robust performance is equivalent with the following, Zhou (1996):

$$
\left\|\mathcal{F}_{u}(M, \Delta)\right\|_{\infty}<1
$$

\section{SIMULATION RESULTS}

The frequency domain analysis (Fig. 5.) showed that the conditions of robust stability (9), nominal performance (10) and robust performance (11) are fulfilled, since all the corresponding norms are smaller than 1 . The achieved $\gamma$ value was 0.0488 , and the designed controller proved to be stable since its poles are on the left-half plane. Consequently, the designed controller is suitable for practical applications. The dominant singular values of the closedloop system have shown that the entire system could be modeled with a second order system (Szeles (2012)).

The time domain analysis showed that control input saturation is necessary, since the magnitude of the controller output was not acceptable physiologically. The simulation was carried out in case of different saturation levels from different initial tumor volumes. In Fig. 6 one can see that tumor volume reaches a steady-state point during the treatment, which attained by the ongoing treatment is independent of the tumor volume at which therapy initiated, and depends only on how the ongoing treatment modulates the balance between angiogenic stimulaters and inhibitors.

At the beginning of the treatment the inhibitor serum level holds a higher constant value, after a while decreases to a lower constant value. The higher the maximal possible serum level of endostatin, the sooner the reduction of the dose is possible (Fig. 7).

Beside maximizing the efficiency of the treatment, another challenge is to minimize the caused strain on the body and the costs of the treatment. The aim of this assess is to show the variation of the total inhibitor inlet as a function of the initial tumor volume and the maximal endostatin inlet. In Fig. 8 one can see how total inhibitor inlet varies with the initial tumor volume.

Higher saturation level can be beneficial in case of fast tumor regression. However, higher inhibitor serum level infers higher total inlet of endostatin, which has to be taken in consideration while planning the therapy. 


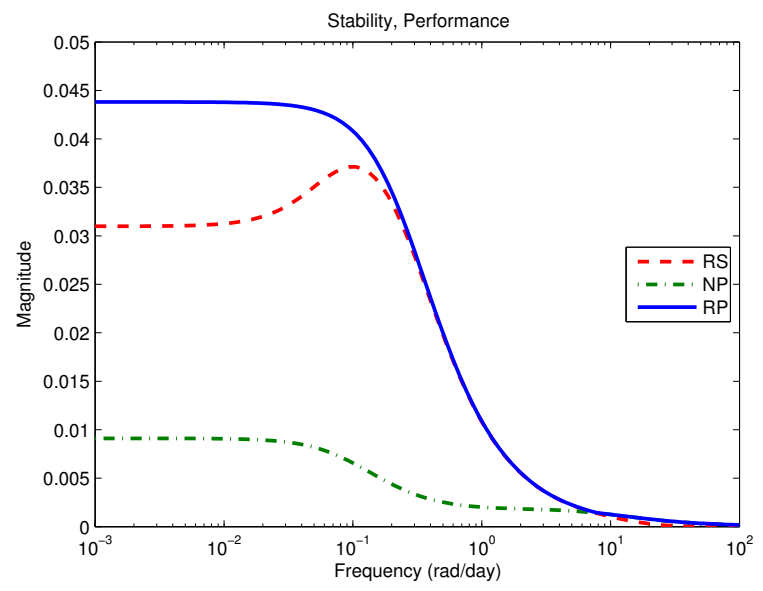

Fig. 5. Robust stability, nominal and robust performance
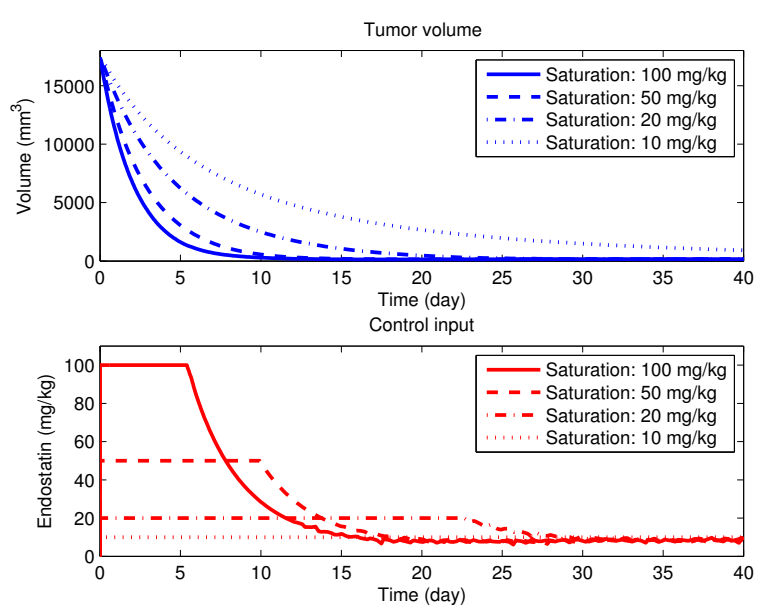

Fig. 6. Tumor regression under control - initial tumor volume $17340 \mathrm{~mm}^{3}$

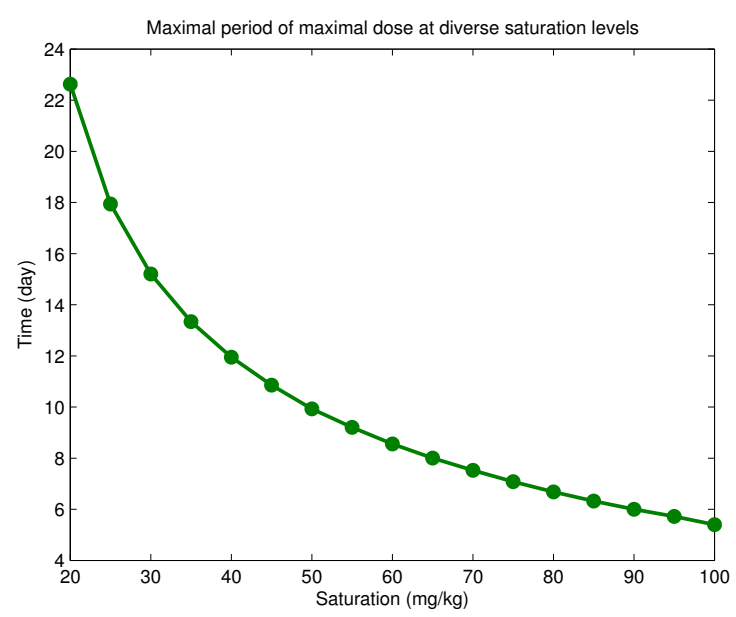

Fig. 7. The period of the maximal constant dose at diverse saturation levels

This paragraph investigates the properties of the closedloop system if the tumor growth model is linearized at diverse working points and the controller design is performed on the latter models, as nominal models. The set point attained by the ongoing treatment and the

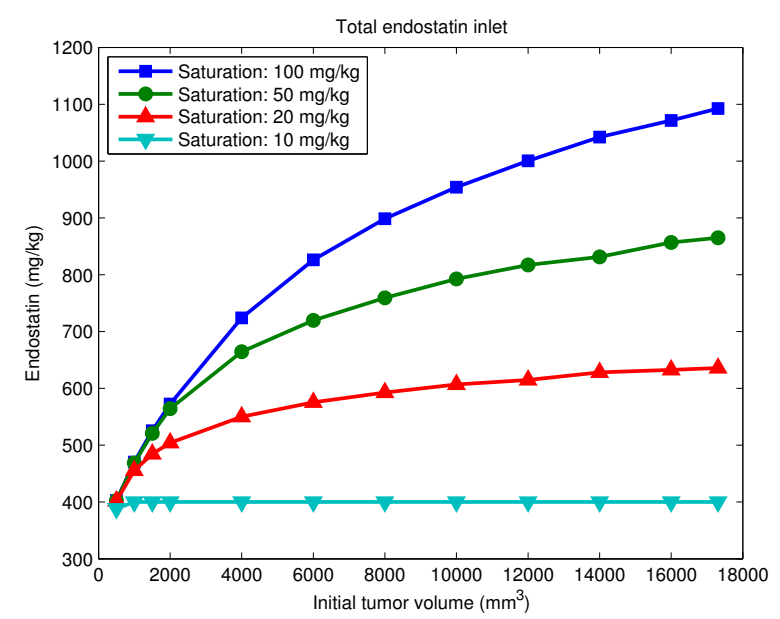

Fig. 8. Inhibitor inlet during the entire treatment

constant inhibitor serum level necessary to retain the tumor volume at a lower level are functions of the working point of the linearization (Fig. 9). The higher the working point of the linearization, the higher the plateau attained and the lower the angiogenic inhibitor serum level required to obtain balance between angiogenic stimulaters and inhibitors.

A controller, which is able to switch between different working points, could achieve good performance at smaller working points, though it could not significantly exceed the performance of the controller presented in the entire domain of interest. Additionally, at larger working points strong deterioration can be expected. Controllers designed at larger operation points show deficiency in diminution of tumor volume, because the magnitude of the control signal is notably limited, (Fig. 10).
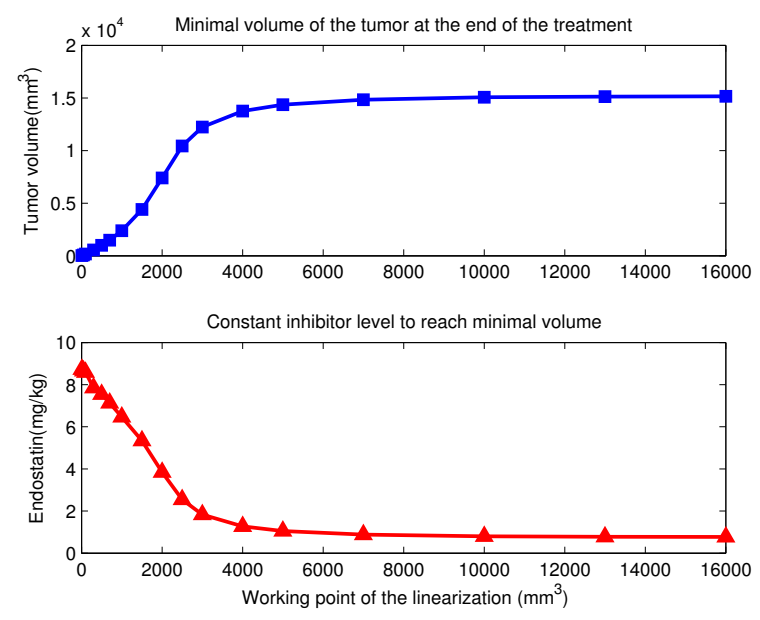

Fig. 9. Minimal tumor volume attained by the treatment and control input required at the plateau

Consequently, good performance, fast tumor regression and small final tumor size can be achieved, if the controller design is performed on a nominal model linearized at small working point, and the controller output is weakly saturated $(50-100 \mathrm{mg} / \mathrm{kg})$. From physiological aspect, moderately saturated $(20-30 \mathrm{mg} / \mathrm{kg})$ inhibitor level is reasonable. Hence, the duration of the treatment is not extreme long, 


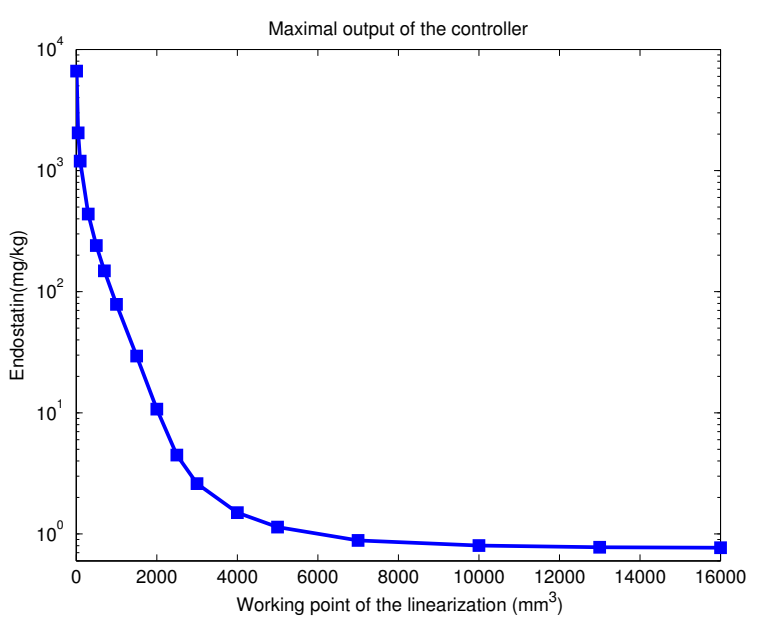

Fig. 10. Maximal output of the controller at diverse working points

and neither the inhibitor serum level nor the total inlet exceeds an acceptable limit.

\section{CONCLUSION}

In the course of $\mathcal{H}_{\infty}$ design robust stability, nominal performance and robust performance were achieved. The properties of the closed-loop system were investigated varying the tumor volume at which treatment was initiated, and at which the nominal model, used by the controller design, was linearized. Tumor regression and total inhibitor inlet was assessed at different saturation levels of the controller output. The controllers, designed at small working points, showed robustness of high degree against the nonlinearities of the system. Good performance, fast tumor regression and small final tumor size were achieved.

Further work will be related to $\mu$-synthesis design, but also on the use of nonlinear control methods and optimal control (Pontryagin (1962), Precup and Preitl (2004)). Higher order model synthesis will be also performed. A further aim of research is modeling and controlling combined therapy and validate it on animal experiments.

\section{REFERENCES}

Doyle, J.C., Glover, K., Khargonekar, P.P., and Francis, B.A. (1989). State-space solutions to standard $\mathcal{H}_{2}$ and $\mathcal{H}_{\infty}$ control problems. IEEE Transactions on Automatic Control, 34(8), 831-847.

Drexler, D.A., Kovács, L., Sápi, J., Harmati, I., and Benyó, Z. (2011). Model-based analysis and synthesis of tumor growth under angiogenic inhibition: a case study. Proc. of the 18th World Congress of the International Federation of Automatic Control, Milano, Italy, 2107, 3753-3758.

Ellis, L.M. and Haller, D.G. (2008). Bevacizumab beyond progression: Does this make sense? Journal of Clinical Oncology, 26(33), 5313-5315.

Gotink, K.J. and Verheul, H.M.W. (2010). Anti-angiogenic tyrosine kinase inhibitors: what is their mechanism of action? Angiogenesis, 13, 1-14.

Gu, D.W., Petkov, P.H., and Konstantinov, M.M. (2005). Robust Control Design with MATLAB. Springer, London.
Hahnfeldt, P., Panigrahy, D., Folkman, J., and Hlatky, L. (1999). Tumor development under angiogenic signaling: A dynamical theory of tumor growth, treatment response, and postvascular dormancy. Cancer Research, 59, 4770-4775.

Holland, J.F. and Frei, E. (2003). Cancer Medicine. BC Decker Inc., Hamilton, Ontario, 6 edition.

Kerbel, R. (1997). A cancer therapy resistant to resistance. Nature, 390, 335-336.

Kleinsmith, L.J., Kerrigan, D., J., K., and Hollen, B. (2010). Understanding Angiogenesis (National Cancer Institute). http://cancer.gov/cancertopics/understandingcancer/ angiogenesis.

Kopper, L. and Tímár, J. (2007). Molecular oncology (in Hungarian). Semmelweis Press.

Kovács, L., Kulcsár, B., György, A., and Benyó, Z. (2011). Robust servo control of a novel type 1 diabetic model. Optimal Control Applications and Methods, 32, 215-238.

Li, J., Chen, F., Conna, M., Feng, Y., Himmelreich, U., Oyen, R., Verbruggen, A., and Ni, Y. (2012). A review on various targeted anticancer therapies. Targeted $\mathrm{On}$ cology.

Liu, W. and Tang, F. (2008). Modeling a simplified regulatory system of blood glucose at molecular levels. Journal of Theoretical Biology, 252, 608-620.

O'Reilly, M.S., Boehm, T., Shing, Y., Fukai, N., Vasios, G., Lane, W.S., Flynn, E., Birkhead, J.R., Olsen, B.R., and Folkman, J. (1997). Endostatin: An endogenous inhibitor of angiogenesis and tumor growth. Cell, 88, 277-285.

Pluda, J.M. (1997). Tumor-associated angiogenesis: mechanisms, clinical implications, and therapeutic strategies. Seminars in Oncology, 24(2), 203-218.

Pontryagin, L.S. (1962). Mathematical Theory of Optimal Processes. Interscience Publishers.

Precup, R.E. and Preitl, S. (2004). Optimisation criteria in development of fuzzy controllers with dynamics. Engineering Applications of Artificial Intelligence, 17(6), $661-674$.

Qian, Y.Y., Zhang, H., Hou, Y., Yuan, L., Li, G.Q., Guo, S., Tadashi, H., and Liu, Y.Q. (2011). Celastrus orbiculatus extract inhibits tumor angiogenesis by targeting vascular endothelial growth factor signaling pathway and shows potent antitumor activity in hepatocarcinomas in Vitro and in Vivo. Chinese Journal of Integrative Medicine, 1-9.

Szeles, A. (2012). Angiogenic Inhibition of Tumor Growth using Modern Robust Control Methods. Master's thesis, Budapest University of Technology and Economics.

The Free Dictionary (2012). Medical dictionary. http://medical-dictionary.thefreedictionary.com/.

World Health Organization (2012). Cancer. http://www.who.int/cancer/en/.

Zhou, K. (1996). Robust and Optimal Control. PrenticeHall, New Jersey. 